\title{
A CRISPR RNA Is Closely Related With the Size of the Cascade Nucleoprotein Complex
}

\author{
Do-Heon Güt, Sung Chul Ha ${ }^{2 * t}$ and Jeong-Sun Kim ${ }^{1 *}$ \\ ${ }^{1}$ Department of Chemistry, Chonnam National University, Gwangju, South Korea, ${ }^{2}$ Pohang Accelerator Laboratory, Pohang, \\ South Korea
}

OPEN ACCESS

Edited by:

Shihui Yang,

Hubei University, China

Reviewed by:

Yibei Xiao,

China Pharmaceutical University,

China

Shan Wu,

Hubei University, China

${ }^{*}$ Correspondence:

Sung Chul Ha

scha2@postech.ac.kr

Jeong-Sun Kim

jsunkim@chonnam.ac.kr

${ }^{t}$ These authors have contributed

equally to this work

Specialty section:

This article was submitted to Microbiotechnology, Ecotoxicology and Bioremediation,

a section of the journal

Frontiers in Microbiology

Received: 27 August 2019 Accepted: 14 October 2019 Published: 29 October 2019

Citation:

Gu D-H, Ha SC and Kim J-S (2019) A CRISPR RNA Is Closely Related With the Size of the Cascade

Nucleoprotein Complex.

Front. Microbiol. 10:2458 doi: 10.3389/fmicb.2019.02458
The currently known prokaryotic adaptive immune system against mobile genetic elements is based on clustered regularly interspaced short palindromic repeats (CRISPR). CRISPR-associated (Cas) proteins and the transcribed short CRISPR RNA (crRNA) molecule form a heterologous ribonucleoprotein complex that neutralizes invading foreign nucleic acids, wherein the crRNA molecule base-pairs with the exogenous genetic elements. In the ribonucleoprotein complexes of the type I CRISPR system, a helical backbone of six identical subunits is commonly found. However, it is not clear how this ribonucleoprotein complex is assembled and what is the determinant factor for its size. We elucidated the crystal structure of the Csy3 subunit of the type I-F ribonucleoprotein complex from Zymomonas mobilis (ZmCsy3), in which seven ZmCsy3 protomers in the asymmetric unit form a molecular helix that is part of a filamentous structure in the entire crystal system. This ZmCsy3 helical structure is remarkably similar to the crRNA-bound hexameric Csy3 backbone from Pseudomonas aeruginosa, with conserved interactions between neighboring subunits. The monomeric ZmCsy3 in solution is transformed into different oligomeric states depending on the added crRNAs. These results suggest that a crRNA and Csy3 subunit play a determinant role in the stepwise formation of the functional Cascade ribonucleoprotein complex and the recruitment of other subunits, and crRNA functions as a molecular ruler for determining the size of the Cascade silencing complex.

Keywords: CRISPR, cascade, Csy3, helical backbone, crRNA

\section{INTRODUCTION}

Many prokaryotes protect themselves from incoming mobile genetic elements using various defense systems (Westra et al., 2012). The clustered regularly interspaced short palindromic repeat (CRISPR)/CRISPR-associated (Cas) system, which is now classified into six types and has been developed as a gene-editing tool, uses the fragments of past visitors that have been inserted into the CRISPR array for recognition of the re-entering genetic elements (Barrangou et al., 2007; Sapranauskas et al., 2011; Huo et al., 2014, PDB ID 4QQW; Gong et al., 2014, PDB ID 4Q2D; Plagens et al., 2015; Amitai and Sorek, 2016). This CRISPR/Cas system removes invading foreign nucleic acids through three stages: (1) in the acquisition step, a portion of the foreign nucleic acids is selectively included in the CRISPR array; (2) the CRISPR array is transcribed as a single long transcript and processed into several mature small CRISPR RNAs (crRNAs) in the expression 
step; and (3) the re-entering exogenous nucleic acids are removed by a ribonucleoprotein complex, frequently called the silencing complex, during the interference step (Barrangou et al., 2007; Brouns et al., 2008; Carte et al., 2008; Hale et al., 2009; Garneau et al., 2010; Zhang et al., 2010; Deltcheva et al., 2011; Jore et al., 2011; Gasiunas et al., 2012; Nam et al., 2012, PDB ID 4F3M; Spilman et al., 2013; Staals et al., 2013). The ribonucleoprotein silencing complex, which acts during the interference step, is composed of mature crRNA and Cas protein(s). The mature crRNA in the silencing complex has two sequential motifs of a spacer sequence in the middle and two short repeat sequences at the $3^{\prime}$ and $5^{\prime}$ ends (Brouns et al., 2008). The repeat sequences at both ends have, in many cases, secondary structures of a partial duplex at the $5^{\prime}$-end ( $5^{\prime}$-handle) and a hairpin structure at the $3^{\prime}$ end ( $3^{\prime}$-hairpin) (Jore et al., 2011; Jackson et al., 2014, PDB ID 4TVX; Mulepati et al., 2014, PDB ID 4QYZ; Zhao et al., 2014, PDB ID 4U7U). The spacer sequence is related with that of past visitors and forms a hetero-duplex with the nucleic acids of repeat visitors. In contrast to a single protein in the ribonucleoproteins of type II and others (Gasiunas et al., 2012; Zetsche et al., 2015), the type I and type III silencing complexes are composed of a number of hetero polypeptides (Jackson et al., 2014; Mulepati et al., 2014; Zhao et al., 2014; Rouillon et al., 2013; Osawa et al., 2015, PDB ID 3X1L) such that several copies of one component form a protein-helical backbone that provides a binding site for the mature crRNAs and other subunits. These components include Cas7 in the type I system (Jackson et al., 2014; Mulepati et al., 2014; Zhao et al., 2014) and Csm4 and Cmr4 in the type III-A and III-B systems, respectively (Rouillon et al., 2013; Osawa et al., 2015).

In the silencing step of the type I CRISPR systems, the CRISPR-associated complex for antiviral defense (Cascade) silencing complex, composed of Cse1, Cse2, Cas7, Cas5e, and Cas6e, and the Cas3 helicase-nuclease protein work together to form the RNA-mediated DNA loop and to remove the reentering DNA. The type I-E Escherichia coli Cascade, revealed by crystallography (Jackson et al., 2014; Mulepati et al., 2014; Zhao et al., 2014), and the type I-F Pseudomonas aeruginosa Cascade, revealed by electron microscopy (EM) (Chowdhury et al., 2017, PDB ID 5uz9; Guo et al., 2017, PUB ID 6NUD), have common structural features and are composed of a proteinhelical backbone formed by six copies of Cas7 or Csy3, an embedded crRNA in the helical backbone, and other subunits. However, it is not clear how each component of the silencing complex is stoichiometrically assembled. The mechanism of assembly for the protein-helical Cascade backbone structures of Cas7 or Csy3 remains unknown; specifically, whether their backbone formation is mediated by crRNA or pre-assembled even in the absence of crRNA has not been elucidated.

The microorganism Zymomonas mobilis ZM4 has three types of CRISPR systems (Seo et al., 2005). Among them, four genes of Csy1, -2, -3, and -4 (ZmCsy1-4) appear to form a silencing Cascade complex in the type I-F CRISPR systems. Based on the $P$. aeruginosa Cascade complex with a protein-helical backbone of six Csy3s, ZmCsy3 is predicted to form the molecular-helical backbone of the Z . mobilis Cascade complex. In order to provide a clue for the assembly mechanism of the Cascade silencing complex of the type I CRISPR/Cas system, the crystal structure of $\mathrm{ZmCsy} 3$ was determined. Interestingly, this protein forms a filament even in the absence of crRNA, which is same as the Csy3 backbone structure observed in the crRNA-bound type I-F Cascade of P. aeruginosa (Chowdhury et al., 2017; Guo et al., 2017). The monomeric $\mathrm{ZmCsy} 3$ protein forms different oligomers in proportion to the added crRNAs of different lengths in solution. These results, together with the reported data, suggest that crRNA may play a major role in assembly of the Cascade complex and determine its size.

\section{MATERIALS AND METHODS}

\section{Construction of a Recombinant Plasmid and Purification of ZmCsy3}

The Csy3 gene (ZMO0684, Met1-Ser346) has 1041 nucleotides, which encode a polypeptide of 346 residues. The target gene was amplified to construct a recombinant plasmid from $Z$. mobilis ZM4 genomic DNA by polymerase chain reaction (PCR) using primers with two restriction enzymes. The amplified PCR product was treated with the restriction enzymes NcoI (New England Biolabs, Beverley, MA, United States) and XhoI (New England Biolabs, Beverley, MA, United States) and was inserted into the vector pHIS2, a derivative of pET21a (Novagen, Madison, WI, United States) that was designed to translate the protein in a fused state with a $6 \mathrm{xHis}$ and tobacco etch virus (TEV) protease cleavage sequence at the $\mathrm{N}$-terminus. The constructed recombinant plasmid was transformed into Escherichia coli BL21Star(DE3), which was grown in LuriaBertani (LB) media supplemented with $50 \mathrm{mg} \cdot \mathrm{mL}^{-1}$ ampicillin at $291 \mathrm{~K}$. When the optical density at $600 \mathrm{~nm}$ reached 0.3 , the fusion protein was expressed by adding $1.0 \mathrm{mM}$ isopropyl $\beta-\mathrm{D}$ 1-thiogalactopyranoside (IPTG) into the culture media followed by incubation for an additional $18 \mathrm{~h}$ at $291 \mathrm{~K}$. The culture was harvested by centrifugation at 5,000 $\mathrm{g}$ at $277 \mathrm{~K}$. The cell pellet was resuspended in an ice-cold buffer A consisting of $20 \mathrm{mM}$ Tris- $\mathrm{HCl} \mathrm{pH}$ 7.5, $10 \mathrm{mM} \beta$-mercaptoethanol, $500 \mathrm{mM} \mathrm{NaCl}$, and $5 \%(\mathrm{v} / \mathrm{v})$ glycerol, and then disrupted by ultrasonication. Cell debris was removed by centrifugation at $11,000 \mathrm{~g}$ for $30 \mathrm{~min}$. The expressed ZmCsy3 fusion protein was initially bound by His-binding agarose resin (ELPIS, Daejeon, South Korea) and the bound protein was eluted by a $500 \mathrm{mM}$ imidazole gradient in buffer A. The eluted ZmCsy3 protein was incubated with the TEV protease to cleave the $6 \mathrm{xHis}$ at the $\mathrm{N}$-terminus and simultaneously dialyzed to remove the salt. After dialysis, the added TEV protein was removed by reloading the protein on the His-binding agarose resin (ELPIS, Daejeon, South Korea). For further purification, anion exchange chromatography was performed using the $5 \mathrm{ml}$ HiTrap $^{\mathrm{T} M} \mathrm{Q}$ column (GE Healthcare, Uppsala, Sweden). The bound protein was released by a linear gradient from 0 to $1 \mathrm{M} \mathrm{NaCl}$ in buffer $\mathrm{A}$. The final purified protein was concentrated up to $13 \mathrm{mg} \cdot \mathrm{ml}^{-1}$ in a buffer consisting of $20 \mathrm{mM}$ Tris- $\mathrm{HCl} \mathrm{pH} 7.5$ and $200 \mathrm{mM} \mathrm{NaCl}$ for crystallization. The protein concentration of the purified $\mathrm{ZmCsy} 3$ was determined by taking into consideration of its extinction coefficient $\left(1.035 \mathrm{M}^{-1} \cdot \mathrm{cm}^{-1}\right)$ at $280 \mathrm{~nm}$. 


\section{Crystallization and Structure Determination}

The purified $\mathrm{ZmCsy} 3$ was crystallized using the sitting-drop vapor-diffusion method in 96-well MRC Crystallization Plates (Molecular Dimension, Maumee, OH, United States). Initial crystallization conditions were screened with the sparse-matrix method (Jancarik and Kim, 1991) using commercially available crystallization kits from Hampton Research (Aliso Viejo, CA, United States) and Rigaku Reagents (Bainbridge Island, WA, United States). The protein solution $(1 \mu \mathrm{l})$ at a concentration of $13 \mathrm{mg} \cdot \mathrm{ml}^{-1}$ was mixed with equal volumes of the screen reagents and the resulting drops were equilibrated against $70 \mu \mathrm{l}$ reservoir solution at $22^{\circ} \mathrm{C}$. The initial conditions were optimized with the hanging-drop vapor-diffusion method using the VDX plate (Hampton Research). The best crystals were grown in the presence of $0.1 \mathrm{M}$ Bis-Tris $\mathrm{pH} 6.5$ and $2.0 \mathrm{M}$ ammonium sulfate. For data collection, a single crystal was cryo-protected with the reservoir solution supplemented with $20 \%$ xylitol and flashfrozen directly in a $-173^{\circ} \mathrm{C}$ nitrogen stream using a nylon loop. A data set consisting of 360 frames of X-ray diffraction data were collected on an ADSC Q270 CCD detector at beamline 7A of the Pohang Light Source II (PLS II) at Pohang Accelerator Laboratory (PAL, Pohang, South Korea) using $0.5^{\circ}$ oscillations at a wavelength of $0.9793 \AA$. The data were indexed and scaled with the HKL-2000 software package (Otwinowski and Minor, 1997). The crystallographic data statistics are summarized in Table 1. The structure was determined with the molecular replacement method in the PHENIX package (Adams et al., 2010; phaser) using the Csy3 molecule (PDB ID 5UZ9; Chowdhury et al., 2017) of $P$. aeruginosa as a search model. The seven models identified were refined with the PHENIX package and the resulting electron density was used for manual rebuilding using Coot (Emsley and Cowtan, 2004). The final model was obtained after iterative manual model building and refinement with Coot and the PHENIX package, respectively. The statistics of refinement are shown in Table 1. Molecular model figures were prepared using the PyMol Molecular graphics program (Schrödinger, LLC). The atomic coordinates and structure factors were deposited in the Protein Data Bank using accession code $6 \mathrm{KQR}^{1}$.

\section{Analytic Size Exclusion Chromatography}

The crRNA of $P$. aeruginosa (CUAAGAAAUUCACGGCGG GCUUGAUGUCCGCGUCUACCUGGUUCA CUGCCGUAU

AGGCAG, the spacer sequences underlined) and its short version (UCACGGCGGGCUUGAUGUCCGCGUCUACCU) containing a 30-nucleotide spacer (from +2 to +31 position of crRNA) were synthesized (Bioneer, South Korea). Each crRNA was mixed with ZmCsy3 at a protein to crRNA ratio of 11 to 1 and 5 to 1 , respectively, and incubated for more than $2 \mathrm{~h}$ at $22^{\circ} \mathrm{C}$. The mixtures were analyzed with a Superdex 200 10/30 column (GE Healthcare) equilibrated with $20 \mathrm{mM}$ Tris pH 7.5, $150 \mathrm{mM}$ $\mathrm{NaCl}$, and $5 \%$ glycerol. The molecular weight of the eluates was estimated based on the positions of known molecular-weight

\footnotetext{
${ }^{1}$ http://www.rcsb.org/pdb
}

TABLE 1 | Data collection and refinement statistics for ZmCsy3.

\begin{tabular}{|c|c|}
\hline Statistics & Se-Met ZmCsy3 \\
\hline \multicolumn{2}{|l|}{ Data collection } \\
\hline Space group & $\mathrm{P} 1$ \\
\hline \multicolumn{2}{|l|}{ Cell dimensions $(\AA)$} \\
\hline$a, b, c(\AA ̊)$ & $82.18,116.16,115.94$ \\
\hline$\alpha, \beta, \gamma\left(^{\circ}\right)$ & $99.36,103.12,103.3590$ \\
\hline Resolution (Å) & $30.0-2.9(2.95-2.9)$ \\
\hline $\mathrm{R}_{\text {merge }}{ }^{\mathrm{a}}(\%)$ & $6.7(46.9)$ \\
\hline $\mathrm{CC}_{1 / 2} \mathrm{~b}^{\mathrm{b}}$ & $0.942(0.745)$ \\
\hline $1 / \sigma(I)$ & $16.0(2.0)$ \\
\hline Completeness (\%) & $98.4(97.9)$ \\
\hline Redundancy & $1.9(1.9)$ \\
\hline Wilson B factor $\left(\AA^{2}\right)$ & 56.92 \\
\hline \multicolumn{2}{|l|}{ Structure refinement } \\
\hline Resolution $(\AA ̊)$ & $30.0-2.9$ \\
\hline Reflections, total/test set & $86047 / 3438$ \\
\hline$R_{\text {work }}{ }^{b} / R_{\text {free }}$ & $19.2(32.5) / 25.9(47.1)$ \\
\hline No. atoms, protein/water & $18907 / 763$ \\
\hline \multicolumn{2}{|l|}{ R.m.s. deviation } \\
\hline Bond lengths $(\AA)$ & 0.008 \\
\hline Angles $\left(^{\circ}\right)$ & 1.015 \\
\hline \multicolumn{2}{|l|}{ Average B-factor $\left(\AA^{2}\right)$} \\
\hline Protein/water & $81.5 / 71.2$ \\
\hline \multicolumn{2}{|l|}{ Ramachandran plot (\%) } \\
\hline Favored region & 91.47 \\
\hline Allowed region & 6.42 \\
\hline
\end{tabular}

The numbers in parentheses are statistics from the highest resolution shell. ${ }^{a} R_{\text {merge }}=\Sigma$ |lobs $-l_{\text {avg }} V I_{\text {obs }}$, where $I_{\text {obs }}$ is the observed intensity of individual reflection and lavg is the average over symmetry equivalents. ${ }^{b}$ Karplus and Diederichs (2012). ${ }^{c} R_{\text {work }}=\Sigma\left\|F_{o}|-| F_{c}\right\| \Sigma \Sigma\left|F_{o}\right|$, where $\left|F_{o}\right|$ and $\left|F_{c}\right|$ are the observed and calculated structure factor amplitudes, respectively. Rfree was calculated with $4 \%$ of the data.

proteins (ferritin, $440 \mathrm{kDa}$; aldolase, $158 \mathrm{kDa}$; ovalbumin, $44 \mathrm{kDa}$; carbonic anhydrase, $29 \mathrm{kDa}$; ribonuclease A, $13.7 \mathrm{kDa}$ ).

\section{RESULTS}

\section{Structural Features of Zymomonas Csy3}

The crystal structure of Csy3 from Z. mobilis ZM4 (ZmCsy3, PDB ID $6 \mathrm{KQR}$ ) was determined by the molecular replacement method at a resolution of $2.9 \AA$ using the Csy 3 component structure of the $P$. aeruginosa Cascade complex (PDB IDs 5UZ9 and 6B44) as a search model (Chowdhury et al., 2017; Guo et al., 2017). There are seven ZmCsy3 molecules (ZmCsy3.1-ZmCsy3.7) in the asymmetric unit, and their structures superimpose very well onto each other, with a root-mean-square-deviation (rmsd) value of less than $0.2 \AA$. The refined structure lacks the first 9 residues at the $\mathrm{N}$-terminus (Met1-Thr9). The monomeric $\mathrm{ZmCsy} 3$ structure (Figure 1A) is composed of ten $\alpha$-helices ( $\alpha 1$ $\alpha 10)$ and eight $\beta$-strands ( $\beta 1-\beta 8)$ and forms a right-handed glove that is composed of a finger of several $\alpha$-helices, a palm consisting of a $\beta$-sheet of $6 \beta$-strands with an extended region (web), a thumb composed of a two-stranded $\beta$-sheet and an $\alpha$-helix, 

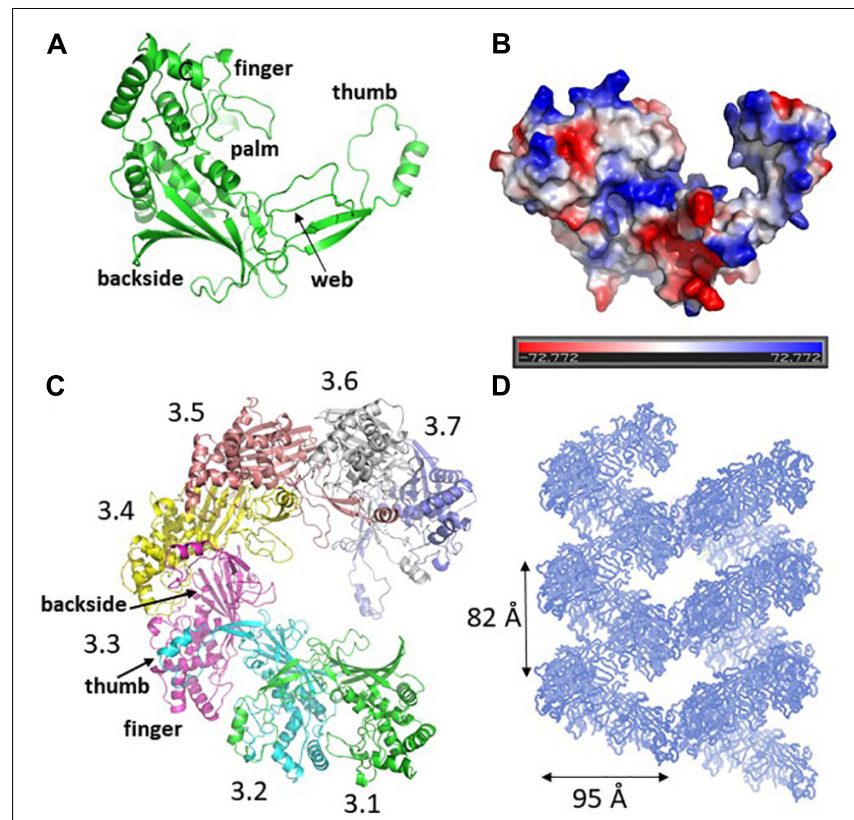

FIGURE 1 | Crystal structure of ZmCsy3. (A) The monomeric structure of ZmCsy3 is presented as a ribbon diagram. Each structural motif is indicated. (B) Surface potential map of the ZmCsy3 molecule in (A). The surface electrostatic potential was calculated using the APBS server (http://www.poissonboltzmann.org/) and is visualized as a color ramp from blue (positive) to red (negative). This figure was rotated with respect to (A). (C) The molecular helix formed by seven Csy3 molecules in the asymmetric unit. Each molecule is differentiated by colors and labeled from 3.1 to 3.7 to indicate the first to 7 th Csy3 molecules. (D) The filamentous structures formed by Csy3 molecules. The symmetry-related molecules are displayed with coils.

and the backside of glove. The surface potential map reveals a positively charged surface patch within the glove and the inner sides of a finger and the thumb, and negatively charged surface patches on the backside of the glove and the outside of a finger and a thumb (Figure 1B).

The seven $\mathrm{ZmCsy} 3$ molecules in the asymmetric unit form an inter-molecular helical structure (Figure 1C). At the intersubunit interface, a thumb of one subunit interacts with a finger of the neighboring subunit, and the $\beta$-sheet of the five $\beta$-strands on the backside of one subunit comes into contact with those of the neighboring subunit(s) through many polar and hydrophilic interactions. In this oligomeric structure, the concave palms are twisted within a single molecule and form a continuous hollow cleft along the molecular helix, together with the extended regions (webs) and several loops in the fingers, and expose a long positively charged cleft to the solvent.

The inter-molecular helix formed by seven ZmCsy3 molecules is part of a long superhelical filament structure in the entire crystal system, where seven molecules form a single turn with a diameter of $\sim 95 \AA$ and a length of $\sim 82 \AA$ (Figure 1D). Since this molecular-helical structure is formed in the absence of crRNA in the crystalline state, there is a possibility that ZmCsy3 can be aggregated at high protein concentrations to form a molecular helical backbone of the Cascade effector complex without crRNA.
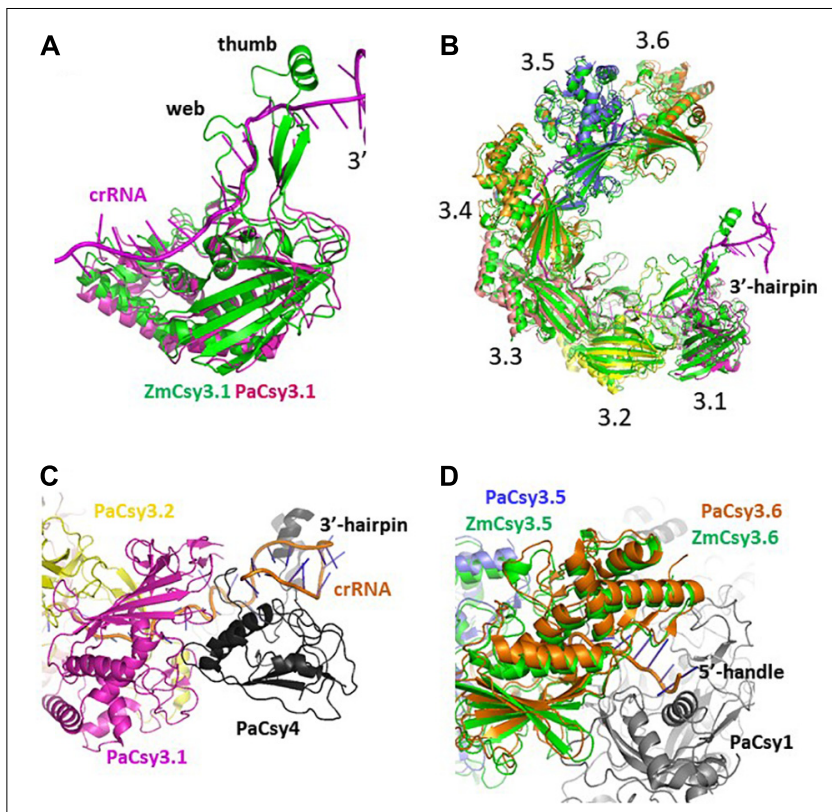

FIGURE 2 | Comparison of Csy3 subunits in the apo- and crRNA-bound Cascade backbone. (A) Superimposed structure of the two monomeric Csy3 structures from $Z$. mobilis and $P$. aeruginosa. The two Csy3 structures are differentiated with colors and the crRNA bound to the PaCsy3 is displayed with a ribbon diagram. (B) The superimposed hexameric Csy Cascade backbone. The ZmCsy3 molecules are displayed in green, while the PaCsy3 molecules are shown with alternating colors. The crRNA bound to the PaCsy3 backbone is displayed as a ribbon. Each Csy3 molecule is labeled from 3.1 at the $3^{\prime}$-hairpin to 3.6 at the $5^{\prime}$-handle. (C) A close-up view of the subunit interaction at the $3^{\prime}$-hairpin region. The $3^{\prime}$-hairpin-interacting PaCsy 4 subunit in the $P$. aeruginosa Cascade complex is displayed as a black ribbon. (D) Close-up view of the subunit interaction at the $5^{\prime}$-handle region. The 5 '-handle-interacting PaCsy1 subunit in the $P$. aeruginosa Cascade complex is displayed as a black ribbon.

\section{Comparison With Other Cascade Backbones}

There are two Cys3 proteins, including the ZmCsy3 studied here, whose structures have been reported (Figure 2; Chowdhury et al., 2017; Guo et al., 2017). ZmCsy3 and P. aeruginosa Csy3 (PaCsy3) have a high sequence identity of $\sim 60 \%$ with some gaps in three regions of the aligned sequences: one is located in the backside of the glove motif and the other two regions are found in the extended loop of the palm motif in the monomeric Csy3 structures (Supplementary Figure S1). The revealed monomeric ZmCsy3 structure superimposes well onto the PaCsy3 structure with rmsd values of less than $1.5 \AA$ among the aligned $325 \mathrm{Ca}$ atoms out of $\sim 340$ residues (Figure 2A). The largest deviations are found in the thumb and the web extended from the palm. Since these deviated regions interact with the bound crRNA in the PaCsy3 of the Pseudomonas Cascade-crRNA complex (Chowdhury et al., 2017; Guo et al., 2017), it is likely that these flexible regions re-orient according to the accessing crRNA.

In the known type I CRISPR Cascade silencing complexes (Jackson et al., 2014; Mulepati et al., 2014; Zhao et al., 2014; Hochstrasser et al., 2016; Chowdhury et al., 2017; Guo et al., 2017; 
Xiao et al., 2017, PDB ID 5U07; Xiao et al., 2018, PDB ID 6C66), six Cas7 or Csy3 molecules are aggregated into a molecular helical structure, which serves as a docking site for crRNA and other Cascade components. Interestingly, the elucidated molecular helix formed by seven ZmCsy3s without crRNA is strikingly similar to the helical backbone of the hexameric PaCsy 3 and EcCas7 (Figure 2). The hexameric molecular-helix formed by ZmCsy3s, except for one ZmCsy3 subunit at either end in the apo-backbone structure, superimposes well onto the PaCsy 3 helix backbone in the holo-Cascade structure, with rmsd values of less than $2.51 \AA$ among the aligned $1912 \mathrm{Ca}$ atoms, and only a negligible difference at the crRNA binding regions (Figure 2B). These structural features strongly indicate that the backboneforming proteins of the type I Cascade complex might selfassemble before binding crRNA and other subunits.

\section{The Monomeric ZmCsy3 Can Form Oligomeric Structures With crRNA in Solution}

Because the observed molecular helix formed by six $\mathrm{ZmCsy} 3$ subunits in the crystalline state closely resembles the molecular helix of PaCsy3 in the holo-Cascade complex (Figure 2), we performed gel-filtration analysis to verify the oligomeric state of ZmCsy3 in solution using the purified ZmCsy3 protein in the presence and absence of various crRNAs.

The gel filtration assays revealed that the purified $\mathrm{ZmCsy} 3$ protein of $1 \mathrm{mg} / \mathrm{ml}$ migrates as a monomer in the absence of crRNA (Figure 3A), while a ZmCsy3 protein of $10 \mathrm{mg} / \mathrm{ml}$ eluted a little earlier than that of $1 \mathrm{mg} / \mathrm{ml}$ (Figure 3B). In contrast, recombinant $\mathrm{ZmCsy} 3$ migrated in oligomeric forms when a 60-nucleotide crRNA derived from the CRISPR array of $P$. aeruginosa, which is expected to bind with six Csy3 molecules, was added into the ZmCsy3 protein solution. Interestingly, adding a 30-nucleotide spacer ranging from the +2 to +31 position of a 60-nucleotide crRNA, which is supposed to bind with four $\mathrm{ZmCsy} 3$ subunits in a sequence-independent manner, also transformed the monomeric $\mathrm{ZmCsy} 3$ into an oligomeric protein. However, the assembled size with the 30-nucleotide spacer crRNA was smaller than with the major oligomer formed by the 60-nucleotide crRNA, indicating that the spacer length of crRNA may be related to the number of recruited ZmCsy3 molecules.

\section{DISCUSSION}

Structural studies of the type I and III silencing effector complexes of the CRISPR systems have often revealed a helical backbone of one subunit that is flanked by different subunits at both ends and provides binding sites for crRNA and other subunits (Jackson et al., 2014; Mulepati et al., 2014; Zhao et al., 2014; Chowdhury et al., 2017; Guo et al., 2017, 2019; Xiao et al., 2017, 2018, PUB ID 6NUD; Jia et al., 2019, PDB ID 6MUR; You et al., 2019, PDB ID 6IFN). In the type I-F system, several Csy genes comprise a silencing Cascade complex with the Csy3 protein as a backbone-forming molecule (Chowdhury et al., 2017; Guo et al., 2017).
Our analysis using gel filtration assay indicated that $\mathrm{ZmCsy3}$ migrates as a monomeric protein at low concentrations (Figure 3A) and does not form a higher oligomeric state at a concentration of $10 \mathrm{mg} / \mathrm{ml}$ (Figure 3B). Nonetheless, there is a high possibility that at high protein concentrations, such as when it is the crystalline state, the Csy3 subunit itself can form an oligomeric structure, which would be very similar to that observed in our current $\mathrm{ZmCsy3}$ oligomeric structure (Figure 2) as well as the Cas7 backbone of the crRNA-bound Cascade complex (Jackson et al., 2014; Mulepati et al., 2014; Zhao et al., 2014; Xiao et al., 2017, 2018). Interestingly, the crRNA-deficient Cmr4 subunit also form a filament structure consisting of eight molecules per turn of $195 \AA$ in the type III-B effector complex (Zhu and Ye, 2015, PDB ID 4WNZ). Even though this Cmr4 filament is similar to that of $\mathrm{ZmCsy3}$, they are rather different in that the ZmCsy3 apo-filament has seven molecules per a single turn with a much shorter distance of $82 \AA$ than that of Cmr4.

The molecular helical backbones formed by Csm 3 subunits in type III-A effector complexes have various copies of the Csm3 subunit (Guo et al., 2019; Jia et al., 2019; You et al., 2019). While two or three Csm3 subunits are observed with a short 36-nucleotide crRNA (Jia et al., 2019; You et al., 2019), five Csm3 copies are observed with a 72-nucleotide crRNA (Guo et al., 2019). Similarly, different numbers of Cas7 subunits have been reported depending on the length of the added crRNA; for example, three Cas7fv in the type I-F in Shewanella putrefaciens with a 43-nucleotide crRNA (Gleditzsch et al., 2016) and three to six Cas7 molecules in E. coli and T. fusca with altered crRNAs (Jackson et al., 2014; Mulepati et al., 2014; Zhao et al., 2014; Kuznedelov et al., 2016; Luo et al., 2016; Xiao et al., 2017). The molecular helical-forming Cmr4 subunits in the type IIIB systems are also found in different numbers (Osawa et al., 2015; Zhu and Ye, 2015). Here, our oligomerization assays with the $\mathrm{ZmCsy} 3$ protein, which exists as a monomeric protein at low concentrations, have shown aggregated $\mathrm{ZmCsy} 3$ proteins of different sizes in proportion to the lengths of the added crRNAs (Figure 3). These features, along with the reported data, strongly suggest that the length of crRNA might be a critical factor for determining the molecular helical backbone size of the silencing complex.

The Cascade silencing complex has a molecular helical backbone of six Cas7 molecules that is flanked by Cas6e and Cas5e at both end, and the former and the latter interact with the $3^{\prime}$-hairpin and the $5^{\prime}$-handle regions of the crRNA, respectively (Jackson et al., 2014; Mulepati et al., 2014; Zhao et al., 2014; Hochstrasser et al., 2016; Xiao et al., 2017, 2018). In this complex structure, the $3^{\prime}$-hairpin structure of crRNA is buried within the protein Cas6e that interacts with Cas7 at one end of the molecular backbone of the Cascade complex, while the $5^{\prime}$-handle region of crRNA is buried within the Cas5e subunit. As mentioned above, crRNA sequences are divided into spacer and repeat sequences, where the relatively well conserved repeat sequence at both ends of a single mature crRNA forms rigid secondary structures, and the variable and elongated spacer sequence in the middle of the crRNA sequence is a multiple of six nucleotides and forms a duplex with exogenous genetic elements through base-pairing. These interactions between the 


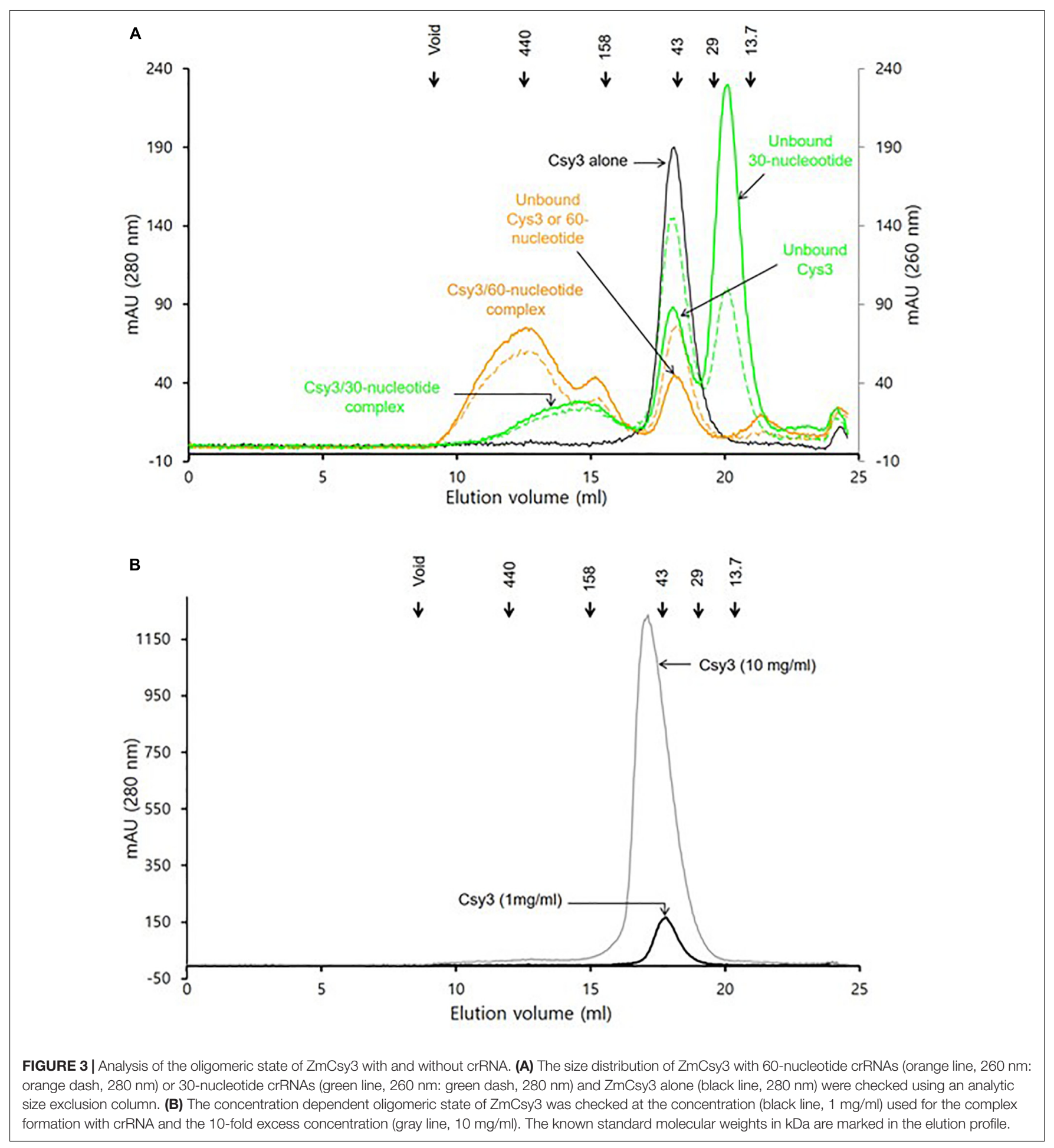

crRNA and the Cascade subunits are also conserved in the type I-F system (Rouillon et al., 2013; Chowdhury et al., 2017; Guo et al., 2017) as well as the type III effector complexes (Osawa et al., 2015). Therefore, the partial secondary structures formed at both ends of crRNA are likely to interfere with the regular interactions between the two molecular helix-forming subunits at the proximal sites and to induce favorable interactions with other Cascade subunits. Our gel filtration assays, conducted with crRNAs that have the same conserved $5^{\prime}$-handle and $3^{\prime}$-hairpin regions but different lengths of spacer sequences, have shown that a small crRNA forms a Csy3 oligomeric protein that is smaller than that formed by a longer crRNA (Figure 3). Taken together, these results suggest that a crRNA with the $3^{\prime}$-hairpin and $5^{\prime}$-handle can be a ruler to guide 
how many molecules should be put together to form the Cascade backbone, and can play a key role in coordinating the assembly of Cascade subunits for the formation of the catalytically active silencing complex, which determines the size of the Cascade, in addition to its well-known function of recognizing exogenous nucleic acids through base-paring between the crRNA and the exogenous nucleic acids.

\section{DATA AVAILABILITY STATEMENT}

The datasets generated for this study can be found in the Protein Data Bank 6KQR.

\section{AUTHOR CONTRIBUTIONS}

J-SK designed the study. All authors performed the experiments, analyzed the data, and wrote the manuscript.

\section{REFERENCES}

Adams, P. D., Afonine, P. V., Bunkóczi, G., Chen, V. B., Davis, I. W., Echols, N., et al. (2010). PHENIX: a comprehensive python-based system for macromolecular structure solution. Acta Crystallogr. D Biol. Crystallogr. 66(Pt 2), 213-221. doi: 10.1107/S0907444909052925

Amitai, G., and Sorek, R. (2016). CRISPR-Cas adaptation: insights into the mechanism of action. Nat. Rev. Microbiol. 14, 67-76. doi: 10.1038/nrmicro. 2015.14

Barrangou, R., Fremaux, C., Deveau, H., Richards, M., Boyaval, P., Moineau, S., et al. (2007). CRISPR provides acquired resistance against viruses in prokaryotes. Science 315, 1709-1712. doi: 10.1126/science.1138140

Brouns, S. J., Jore, M. M., Lundgren, M., Westra, E. R., Slijkhuis, R. J., Snijders, A. P., et al. (2008). Small CRISPR RNAs guide antiviral defense in prokaryotes. Science 321, 960-964. doi: 10.1126/science.1159689

Carte, J., Wang, R., Li, H., Terns, R. M., and Terns, M. P. (2008). Cas6 is an endoribonuclease that generates guide RNAs for invader defense in prokaryotes. Genes Dev. 22, 3489-3496. doi: 10.1101/gad.1742908

Chowdhury, S., Carter, J., Rollins, M. F., Golden, S. M., Jackson, R. N., Hoffmann, C., et al. (2017). Structure reveals mechanisms of viral suppressors that intercept a CRISPR RNA-guided surveillance complex. Cell 169, 47-57.e11. doi: 10.1016/ j.cell.2017.03.012

Deltcheva, E., Chylinski, K., Sharma, C. M., Gonzales, K., Chao, Y., Pirzada, Z. A., et al. (2011). CRISPR RNA maturation by trans-encoded small RNA and host factor RNase III. Nature 471, 602-607. doi: 10.1038/nature09886

Emsley, P., and Cowtan, K. (2004). Coot: model-building tools for molecular graphics. Acta Crystallogr. D Biol. Crystallogr. 60, 2126-2132. doi: 10.1107/ s0907444904019158

Garneau, J. E., Dupuis, M. E., Villion, M., Romero, D. A., Barrangou, R., Boyaval, P., et al. (2010). The CRISPR Cas bacterial immune system cleaves bacteriophage and plasmid DNA. Nature 468, 67-71. doi: 10.1038/nature09523

Gasiunas, G., Barrangou, R., Horvath, P., and Siksnys, V. (2012). Cas9-crRNA ribonucleoprotein complex mediates specific DNA cleavage for adaptive immunity in bacteria. Proc. Natl. Acad. Sci. U.S.A. 109, E2579-E2586.

Gleditzsch, D., Müller-Esparza, H., Pausch, P., Sharma, K., Dwarakanath, S., Urlaub, H., et al. (2016). Modulating the cascade architecture of a minimal type I-F CRISPR-Cas system. Nucleic Acids Res. 44, 5872-5882. doi: 10.1093/nar/ gkw469

Gong, B., Shin, M., Sun, J., Jung, C. H., Bolt, E. L., van der Oost, J., et al. (2014). Molecular insights into DNA interference by CRISPR-associated nucleasehelicase Cas3. Proc. Natl. Acad. Sci. U.S.A. 111, 16359-16364. doi: 10.1073/pnas. 1410806111

\section{FUNDING}

This work was supported by the Basic Science Research Programs (NRF Grant Number: 2017R1D1A3B03032278) of the National Research Foundation (NRF) of South Korea, funded by the Ministry of Education, Science and Technology of Korea (MEST).

\section{ACKNOWLEDGMENTS}

X-ray diffraction experiments were performed with beamline 7A at the Pohang Accelerator in South Korea.

\section{SUPPLEMENTARY MATERIAL}

The Supplementary Material for this article can be found online at: https://www.frontiersin.org/articles/10.3389/fmicb. 2019.02458/full\#supplementary-material

Guo, M., Zhang, K., Zhu, Y., Pintilie, G. D., Guan, X., Li, S., et al. (2019). Coupling of ssRNA cleavage with DNase activity in type III-A CRISPR-Csm revealed by cryo-EM and biochemistry. Cell Res. 29, 305-312. doi: 10.1038/s41422-0190151-X

Guo, T. W., Bartesaghi, A., Yang, H., Falconieri, V., Rao, P., Merk, A., et al. (2017). Cryo-EM structures reveal mechanism and inhibition of DNA targeting by a CRISPR-Cas surveillance complex. Cell 171, 414-426.e12. doi: 10.1016/j.cell. 2017.09.006

Hale, C. R., Zhao, P., Olson, S., Duff, M. O., Graveley, B. R., Wells, L., et al. (2009). RNA-guided RNA cleavage by a CRISPR RNA-Cas protein complex. Cell 139, 945-956. doi: 10.1016/j.cell.2009.07.040

Hochstrasser, M. L., Taylor, D. W., Kornfeld, J. E., Nogales, E., and Doudna, J. A. (2016). DNA targeting by a minimal CRISPR RNA-guided cascade. Mol. Cell 63, 840-851. doi: 10.1016/j.molcel.2016.07.027

Huo, Y., Nam, K. H., Ding, F., Lee, H., Wu, L., Xiao, Y., et al. (2014). Structures of CRISPR Cas3 offer mechanistic insights into Cascade-activated DNA unwinding and degradation. Nat. Struct. Mol. Biol. 21, 771-777. doi: 10.1038/ nsmb. 2875

Jackson, R. N., Golden, S. M., van Erp, P. B., Carter, J., Westra, E. R., Brouns, S. J., et al. (2014). Structural biology. Crystal structure of the CRISPR RNAguided surveillance complex from Escherichia coli. Science 345, 1473-1479. doi: $10.1126 /$ science. 1256328

Jancarik, J., and Kim, S.-H. (1991). Sparse matrix sampling: a screening method for crystallization of proteins. J. Appl. Cryst. 24, 409-411. doi: 10.1107/ S0021889891004430

Jia, N., Mo, C. Y., Wang, C., Eng, E. T., Marraffini, L. A., and Patel, D. J. (2019). Type III-A CRISPR-Cas Csm complexes: assembly, periodic RNA cleavage, DNase activity regulation, and autoimmunity. Mol. Cell. 73, 264-277.e5. doi: 10.1016/j.molcel.2018.11.007

Jore, M. M., Lundgren, M., van Duijn, E., Bultema, J. B., Westra, E. R., Waghmare, S. P., et al. (2011). Structural basis for CRISPR RNA-guided DNA recognition by cascade. Nat. Struct. Mol. Biol. 8, 529-536. doi: 10.1038/nsmb.2019

Karplus, P. A., and Diederichs, K. (2012). Linking crystallographic model and data quality. Science 336, 1030-1033. doi: 10.1126/science.1218231

Kuznedelov, K., Mekler, V., Lemak, S., Tokmina-Lukaszewska, M., Datsenko, K. A., Jain, I., et al. (2016). Altered stoichiometry Escherichia coli Cascade complexes with shortened CRISPR RNA spacers are capable of interference and primed adaptation. Nucleic Acids Res. 44, 10849-10861. doi: 10.1093/nar/gkw914

Luo, M. L., Jackson, R. N., Denny, S. R., Tokmina-Lukaszewska, M., Maksimchuk, K. R., Lin, W., et al. (2016). The CRISPR RNA-guided surveillance complex in Escherichia coli accommodates extended RNA spacers. Nucleic Acids Res. 44, 7385-7394. doi: 10.1093/nar/gkw421 
Mulepati, S., Héroux, A., and Bailey, S. (2014). Structural biology. crystal structure of a CRISPR RNA-guided surveillance complex bound to a ssDNA target. Science 345, 1479-1484. doi: 10.1126/science.1256996

Nam, K. H., Haitjema, C., Liu, X., Ding, F., Wang, H., DeLisa, M. P., et al. (2012). Cas5d protein processes pre-crRNA and assembles into a cascade-like interference complex in subtype I-C/Dvulg CRISPR-Cas system. Structure 20, 1574-1584. doi: 10.1016/j.str.2012.06.016

Osawa, T., Inanaga, H., Sato, C., and Numata, T. (2015). Crystal structure of the CRISPR-Cas RNA silencing Cmr complex bound to a target analog. Mol. Cell. 58, 418-430. doi: 10.1016/j.molcel.2015.03.018

Otwinowski, Z., and Minor, W. (1997). Processing of X-ray diffraction data collected in oscillation mode. Methods Enzymol. 276, 307-326. doi: 10.1016/ S0076-6879(97)76066-X

Plagens, A., Richter, H., Charpentier, E., and Randau, L. (2015). DNA and RNA interference mechanisms by CRISPR-Cas surveillance complexes. FEMS Microbiol. Rev. 39, 442-463. doi: 10.1093/femsre/fuv019

Rouillon, C., Zhou, M., Zhang, J., Politis, A., Beilsten-Edmands, V., Cannone, G., et al. (2013). Structure of the CRISPR interference complex CSM reveals key similarities with cascade. Mol. Cell. 52, 124-134. doi: 10.1016/j.molcel.2013. 08.020

Sapranauskas, R., Gasiunas, G., Fremaux, C., Barrangou, R., Horvath, P., and Siksnys, V. (2011). The Streptococcus thermophilus CRISPR/Cas system provides immunity in Escherichia coli. Nucleic Acids Res. 39, 9275-9282. doi: 10.1093/nar/gkr606

Seo, J. S., Chong, H., Park, H. S., Yoon, K. O., Jung, C., Kim, J. J., et al. (2005). The genome sequence of the ethanologenic bacterium Zymomonas mobilis ZM4. Nat. Biotechnol. 23, 63-68.

Spilman, M., Cocozaki, A., Hale, C., Shao, Y., Ramia, N., Terns, R., et al. (2013). Structure of an RNA silencing complex of the CRISPR-Cas immune system. Mol. Cell. 52, 146-152. doi: 10.1016/j.molcel.2013.09.008

Staals, R. H., Agari, Y., Maki-Yonekura, S., Zhu, Y., Taylor, D. W., van Duijn, E., et al. (2013). Structure and activity of the RNA-targeting type III-B CRISPRCas complex of Thermus thermophilus. Mol. Cell. 52, 135-145. doi: 10.1016/j. molcel.2013.09.013

Westra, E. R., Swarts, D. C., Staals, R. H., Jore, M. M., Brouns, S. J., and van der Oost, J. (2012). The CRISPRs, they are a-changin': how prokaryotes generate adaptive immunity. Annu. Rev. Genet. 46, 311-339. doi: 10.1146/annurevgenet-110711-155447

Xiao, Y., Luo, M., Dolan, A. E., Liao, M., and Ke, A. (2018). Structure basis for RNA-guided DNA degradation by Cascade and Cas3. Science 361:eaat0839. doi: $10.1126 /$ science.aat 0839

Xiao, Y., Luo, M., Hayes, R. P., Kim, J., Ng, S., Ding, F., et al. (2017). Structure basis for directional R-loop formation and substrate handover mechanisms in type I CRISPR-Cas system. Cell 170, 48-60.e11. doi: 10.1016/j.cell.2017.06.012

You, L., Ma, J., Wang, J., Artamonova, D., Wang, M., Liu, L., et al. (2019). Structure studies of the CRISPR-Csm complex reveal mechanism of co-transcriptional interference. Cell 176, 239-253.e16. doi: 10.1016/j.cell.2018.10.052

Zetsche, B., Gootenberg, J. S., Abudayyeh, O. O., Slaymaker, I. M., Makarova, K. S., Essletzbichler, P., et al. (2015). Cpf1 is a single RNA-guided endonuclease of a class 2 CRISPR-Cas system. Cell 163:759. doi: 10.1016/j.cell.2015. 09.038

Zhang, J., Abadia, E., Refregier, G., Tafaj, S., Boschiroli, M. L., Guillard, B., et al. (2010). Mycobacterium tuberculosis complex CRISPR genotyping: improving efficiency, throughput and discriminative power of 'spoligotyping' with new spacers and a microbead-based hybridization assay. J. Med. Microbiol. 59(Pt 3), 285-294. doi: 10.1099/jmm.0.016949-0

Zhao, H., Sheng, G., Wang, J., Wang, M., Bunkoczi, G., Gong, W., et al. (2014). Crystal structure of the RNA-guided immune surveillance cascade complex in Escherichia coli. Nature 515, 147-150. doi: 10.1038/nature13733

Zhu, X., and Ye, K. (2015). Cmr4 is the slicer in the RNA-targeting Cmr CRISPR complex. Nucleic Acids Res. 43, 1257-1267. doi: 10.1093/nar/gku1355

Conflict of Interest: The authors declare that the research was conducted in the absence of any commercial or financial relationships that could be construed as a potential conflict of interest.

Copyright (c) $2019 \mathrm{Gu}, \mathrm{Ha}$ and Kim. This is an open-access article distributed under the terms of the Creative Commons Attribution License (CC BY). The use, distribution or reproduction in other forums is permitted, provided the original author(s) and the copyright owner(s) are credited and that the original publication in this journal is cited, in accordance with accepted academic practice. No use, distribution or reproduction is permitted which does not comply with these terms. 